\title{
Time-Frequency Feature Extraction of Newborn EEG Seizure Using SVD-Based Techniques
}

\author{
Hamid Hassanpour \\ Lab of Signal Processing Research, Queensland University of Technology, GPO Box 2434, Brisbane, QLD 4001, Australia
}

Email: h.hassanpour@qut.edu.au

Mostefa Mesbah

Lab of Signal Processing Research, Queensland University of Technology, GPO Box 2434, Brisbane, QLD 4001, Australia Email:m.mesbah@qut.edu.au

\author{
Boualem Boashash \\ Lab of Signal Processing Research, Queensland University of Technology, GPO Box 2434, Brisbane, QLD 4001, Australia \\ Email: b.boashash@qut.edu.au
}

Received 27 August 2003; Revised 17 May 2004

\begin{abstract}
The nonstationary and multicomponent nature of newborn EEG seizures tends to increase the complexity of the seizure detection problem. In dealing with this type of problems, time-frequency-based techniques were shown to outperform classical techniques. This paper presents a new time-frequency-based EEG seizure detection technique. The technique uses an estimate of the distribution function of the singular vectors associated with the time-frequency distribution of an EEG epoch to characterise the patterns embedded in the signal. The estimated distribution functions related to seizure and nonseizure epochs were used to train a neural network to discriminate between seizure and nonseizure patterns.
\end{abstract}

Keywords and phrases: detection, time-frequency distribution, singular value decomposition, probability distribution function.

\section{INTRODUCTION}

Clinical signs of central nervous system dysfunctions in the neonate are often revealed by seizures which are the results of synchronous discharge of a large number of neurons [1]. Seizures increase the risk of impaired neurological and developmental functioning in neonatal period and also increase the risk of death [2].

Clinical manifestations of seizure in adults such as body jerking, repetitive winking, or fluttering of eyelids are well defined and easily recognisable. However, in newborns, the clinical signs are not as clear and can be missed without constant and close supervision. In neonates, the brain function is constantly changing as its neurophysiology matures $[3,4]$. This emphasises the nonstationary behaviour of the electroencephalogram (EEG) in neonates $[5,6]$. In addition, the frequency spectrum of the background EEG largely overlaps with the seizure one [7]. This behaviour makes the task of analysing newborn EEG signal a complex one for both neurologists and signal analysts. To overcome this complexity, time-frequency- (TF) based techniques were introduced.
Neonatal EEG seizures have signatures in both low frequency (as low as $0.5 \mathrm{~Hz}$ ) [8] and high frequency (higher than $70 \mathrm{~Hz}$ ) [9]. This study concentrates on using the lowfrequency signatures for seizure detection. Detection of EEG seizures using the low-frequency signature requires a lower number of data samples, hence the computational time is reduced. To remove the high-frequency activity, the signal is filtered by a lowpass filter with a cutoff frequency $10 \mathrm{~Hz}$. The filtered signal is then segmented into 30-second epochs. By choosing 30-second epochs we are not assuming that the minimum seizure length is 30 seconds. Indeed, in the presented technique, no limitation for the minimum length of seizure was assumed. However, the longer the duration of EEG epochs, the better is the discrimination between seizures and nonseizures. Choosing 30 seconds for the duration of epochs is found to be adequate for the feature extraction process and also alleviates the computation task. Once the EEG is segmented, the epochs are mapped into the TF domain. To extract the features of the seizure, we use a singularvalue-decomposition- (SVD) based technique applied to the TF distribution (TFD) of the EEG epochs. Singular vectors 
(SVs) of a matrix are the span bases of the matrix, and their importance in the composition of the matrix is reflected by the related singular values. Since SVs are orthonormal [10], their squared elements can be treated as probability density function (PDF) [11]. These PDFs are used in the process of seizure feature extraction in this paper.

\section{EEG DATA ACQUISITION}

EEG data acquisition was performed on the newborn, ages ranging between two days and two weeks, at the Royal Women's Hospital, Brisbane, Australia. The electrodes were placed on the scalp according to the 10-20 International System of Electrode Placement. The data were recorded on 20 channels using Medelec (Oxford Instruments, UK) software/hardware environment. The sampling frequency was set to $256 \mathrm{~Hz}$. The seizure activities on the recordings were visually labeled by a neurologist from the Neurosciences Department at the Royal Children's Hospital.

\section{TF-BASED FEATURE EXTRACTION}

In analysing nonstationary and multicomponent signals, the TF-based techniques have been shown to outperform classical techniques based on either time or frequency domains [12]. SVs of the matrix associated with the TFD of a signal can be used to characterise the signal. By using the SVD technique, the SVs and their importance in the composition of the matrix (singular values) are computed.

\subsection{TFD of signals}

TFDs are powerful tools for extracting features of the patterns embedded in a nonstationary signal [13]. The TFD of a signal is a joint representation in both time and frequency domains. For a given signal $x(t)$, the TFD that belongs to the quadratic class can be expressed as [14]

$$
\begin{array}{r}
\rho_{z}(t, f)=\int_{-\infty}^{\infty} \int_{-\infty}^{\infty} \int_{-\infty}^{\infty} e^{j 2 \pi v(u-t)} g(v, \tau) z\left(u+\frac{\tau}{2}\right) z^{*} \\
\times\left(u-\frac{\tau}{2}\right) e^{-j 2 \pi f \tau} d v d u d \tau,
\end{array}
$$

where $z(t)$ is the analytic signal associated with $x(t)$, and $g(v, \tau)$ is a 2 -dimensional kernel that determines the characteristics of the TFD. For example, by setting $g(v, \tau)=1$, we get the Wigner-Ville distribution (WVD), and with $g(\nu, \tau)=$ $|\tau|^{\beta}|\Gamma(\beta+j \pi v)|^{2} / 2^{1-2 \beta} \Gamma(2 \beta)$, the equation represents the Bdistribution [15]. In the B-distribution, $\Gamma(\cdot)$ represents the Gamma function and $\beta$ is a smoothing parameter.

The bilinear operation on the signal in (1) may produce spurious components, cross-terms, in the TFD when the signal is a multicomponent or a nonlinear FM [16]. The reduced interference distributions (RIDs), such as the Bdistribution, have been introduced to reduce the effect of cross-terms on the TFD of a signal [15]. Different TF kernels are valuable under certain conditions, hence their suitabil- ity is application dependent. It has been shown that the Bdistribution is very suitable, in terms of resolution and crossterms, for analysing the low-frequency activities in the EEG signal [8]. A TFD with high resolution provides a better insight in the analysis of signals, especially when the signals are multicomponent and the components are close to each other.

\subsection{SVD}

The SVD method has been a valuable tool in signal processing and statistical data analysis. A SVD of an $M \times N$ matrix $X$, representing the TFD of the signal $x$, is given by

$$
X=U \Sigma V^{T},
$$

where $U(M \times M)$ and $V(N \times N)$ are orthonormal matrices, and $\Sigma$ is an $M \times N$ diagonal matrix of singular values $\left(\sigma_{i j}=0\right.$ if $i \neq j$ and $\sigma_{11} \geq \sigma_{22} \geq \cdots \geq 0$ ). The columns of the orthonormal matrices $U$ and $V$ are called the left and right SVs, respectively. An important property of $U$ and $V$ is that they are mutually orthogonal [10]. The singular values $\left(\sigma_{i i}\right)$ represent the importance of individual SVs in the composition of the matrix. In other words, SVs corresponding to the larger singular values have more information about the structure of patterns embedded in the matrix than the other SVs.

\subsection{Using SVs to characterise signal in the TF domain}

In the analysis of signals in the TF domain using SVD, the type of TF distribution is important. Indeed, it is desirable that the TFD has both less cross-terms and high resolution. To satisfy these specifications, we use the B-distribution [17], which has been shown to give good performance for newborn EEG signals [8].

Previous researches have mostly concentrated on features based only on the singular values of the TFD of the signals $[18,19]$. By themselves, singular values do not carry significant information about the behaviour of patterns embedded in the matrix. In other words, they are not suitable features for classification purposes $[11,20]$.

To find the characteristics of a signal in the TF domain using the SVD technique, we propose to use the right and left SVs corresponding to the largest singular values. The reason is that the right and left SVs contain the time and frequency domain information of the signal, respectively [11]. In addition, SVs related to the largest singular values have more information about the structure of the signal. Consequently, if the structure of signals are different for dissimilar classes, using SVs related to the largest singular values is more suitable for classification [21]. However, SVs corresponding to the lowest singular values would be more appropriate if the structure of different classes are similar to each other (see, e.g., [22]).

To show that both left and right SVs are necessary to characterise a signal in the TF domain, examples are given below. Assume that $x_{1}(t)$ and $x_{2}(t)$ are two linear FM signals in 


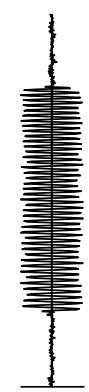

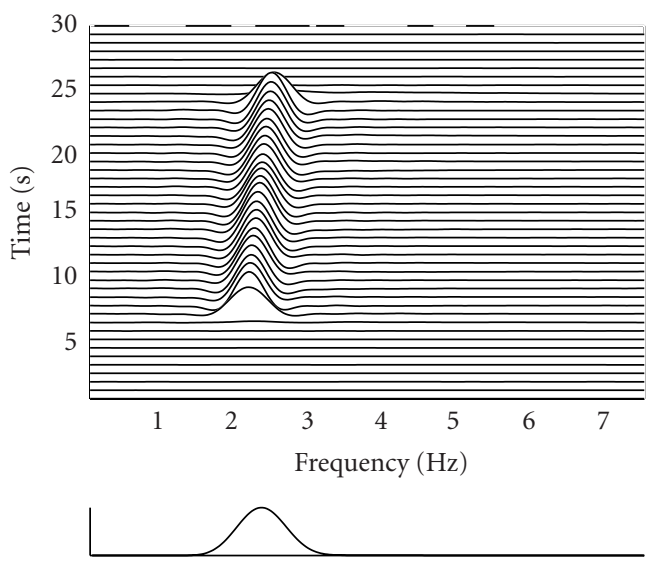

(a)

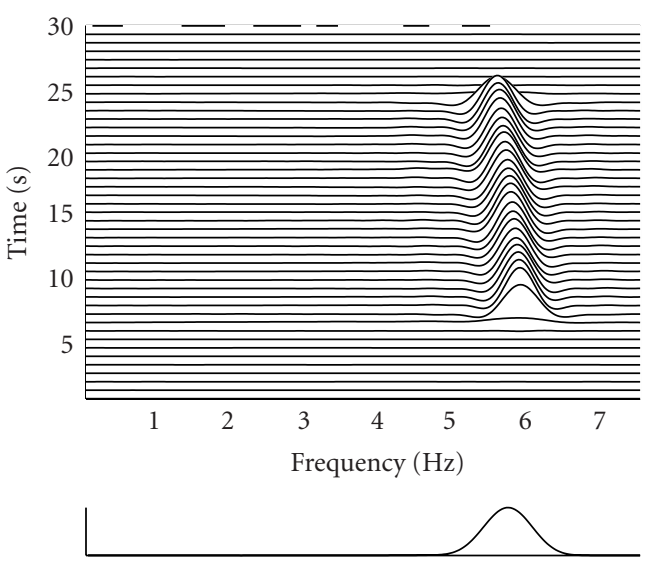

(b)

Figure 1: The TFD of two linear FM signals in the noise: (a) $t_{1}(t)$ and (b) $x_{2}(t)(F s=15 \mathrm{~Hz}, N=450$, time resolution= 5).

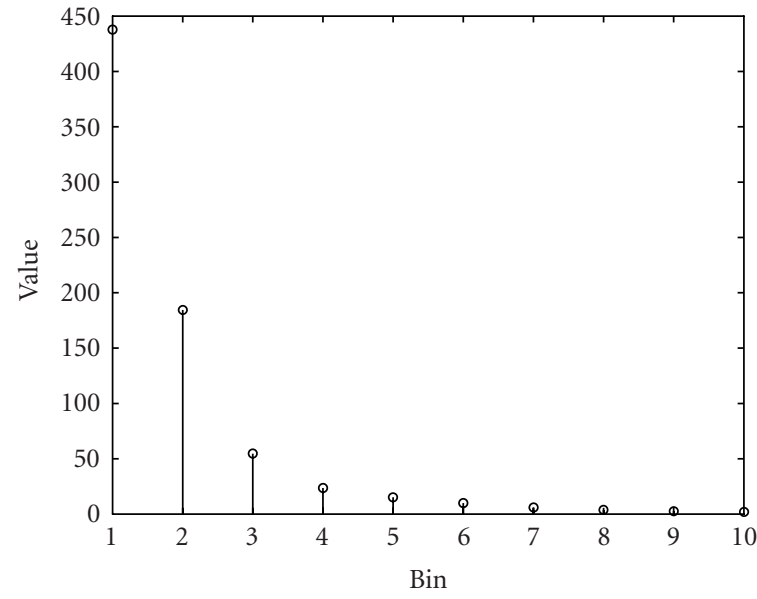

(a)

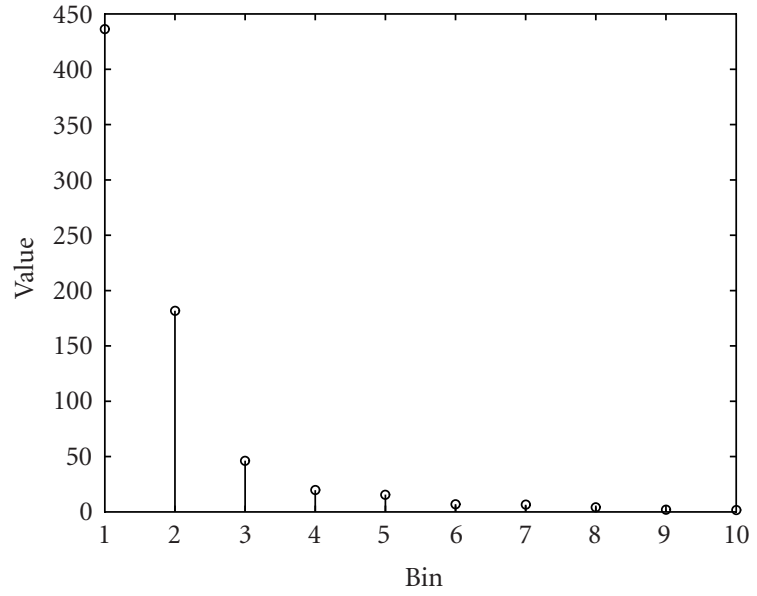

(b)

FIgURE 2: The first ten singular values of the TFDs related to (a) $x_{1}(t)$ and (b) $x_{2}(t)$.

white noise $n(t)$; that is,

$$
\begin{aligned}
& x_{1}(t)=\sin \left(4 \pi t+0.02 \pi t^{2}\right) \Pi\left(\frac{t-15}{18}\right)+n(t), \\
& x_{2}(t)=\sin \left(12 \pi t-0.02 \pi t^{2}\right) \Pi\left(\frac{t-15}{18}\right)+n(t),
\end{aligned}
$$

where $\Pi(\cdot)$ is the rectangular function. The rectangular function is defined as

$$
\Pi(\alpha)= \begin{cases}1 & \text { if }-\frac{1}{2}<\alpha<\frac{1}{2}, \\ 0 & \text { otherwise }\end{cases}
$$

The TFDs of these two signals are shown in Figure 1. In the figure, the power spectral density and the time-domain representation of the signal are displayed at the bottom and left side of the TF plane. The singular values related to the TFD of these signals (see Figure 2) show that the two signals are not distinguishable using the singular values alone. Figure 3 shows the first two right SVs related to the TFDs of Figure 1. These two SVs are similar in spite of the fact that the two signals are different. Figure 4, however, shows that the two left SVs are different.

Another example that illustrates the above claim is given below. Assume that

$x_{3}(t)=\sin \left(4 \pi t+0.02 \pi t^{2}\right)\left(\Pi\left(\frac{t-9}{6}\right)+\Pi\left(\frac{t-21}{6}\right)\right)+n(t)$.

The TFD of the signal along with the singular values and the first two SVs related to the TFD are shown in Figure 5. It can be seen in the figure that the left SVs are similar to those of $x_{1}(t)$ represented in Figure $4 \mathrm{a}$, whereas the right SVs are different. 

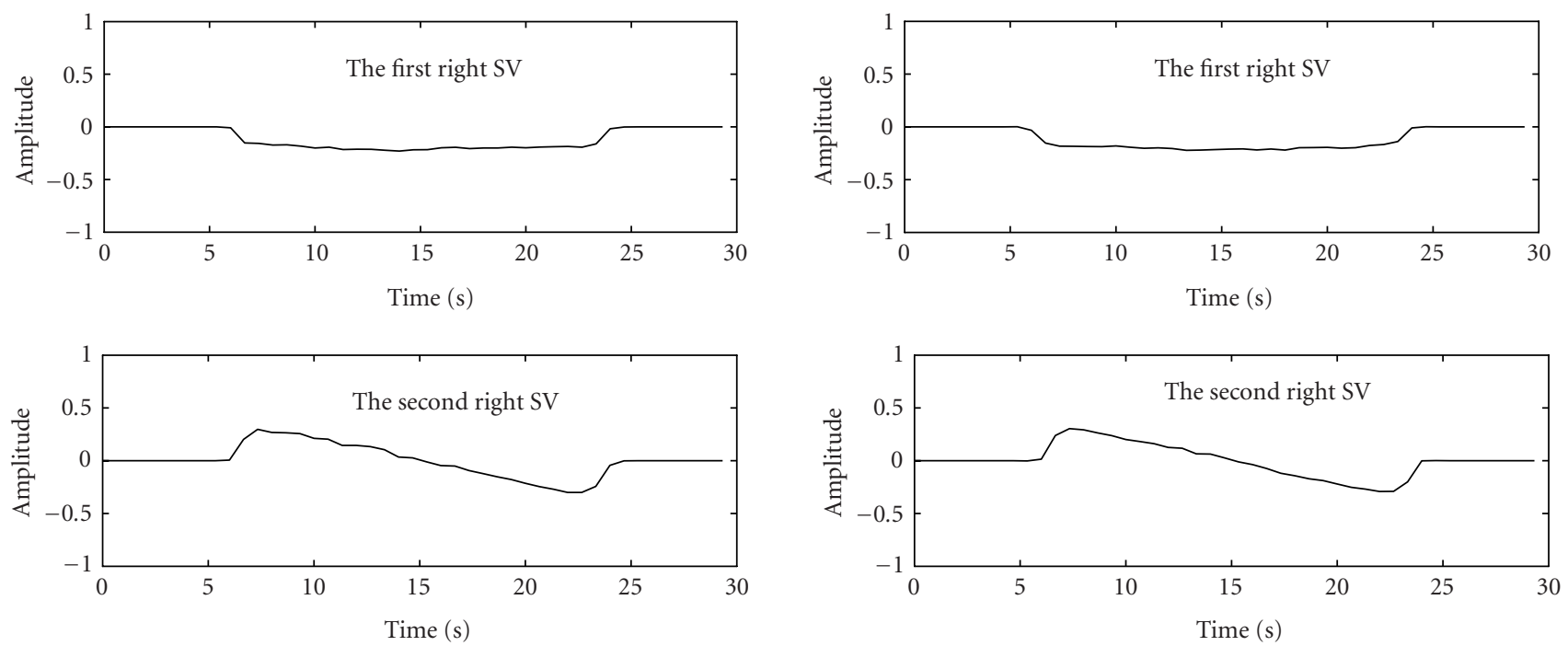

(a)

(b)

FIgure 3: The first two right SVs of the TFDs related to (a) $x_{1}(t)$ and (b) $x_{2}(t)$.
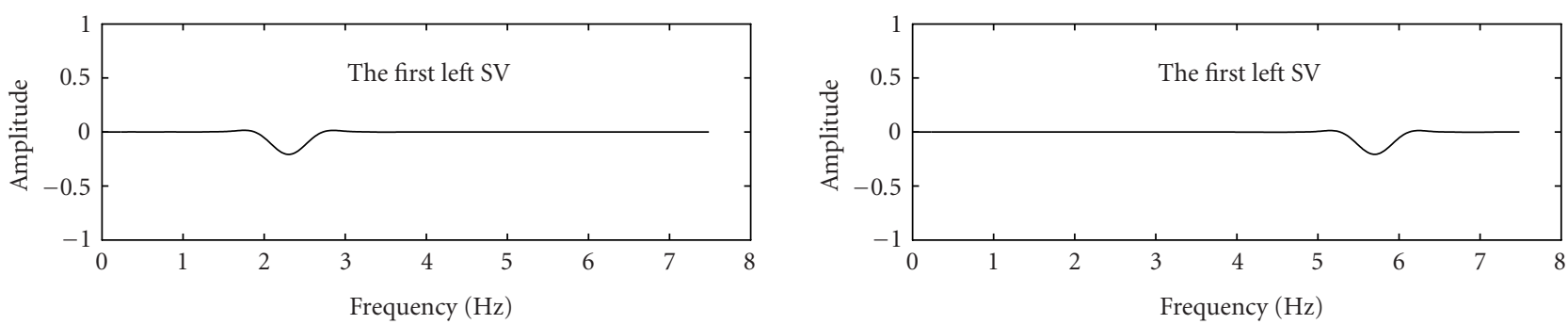

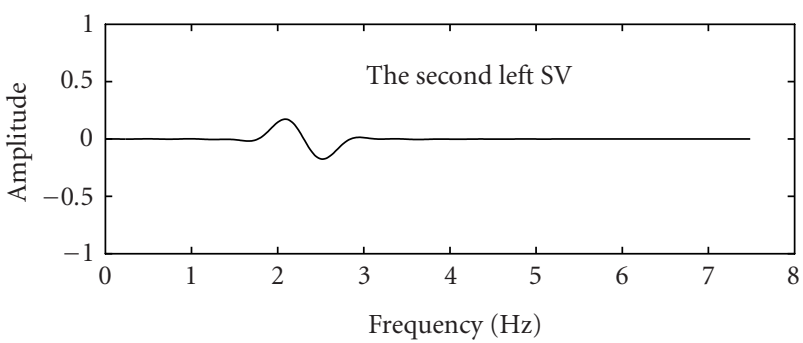

(a)

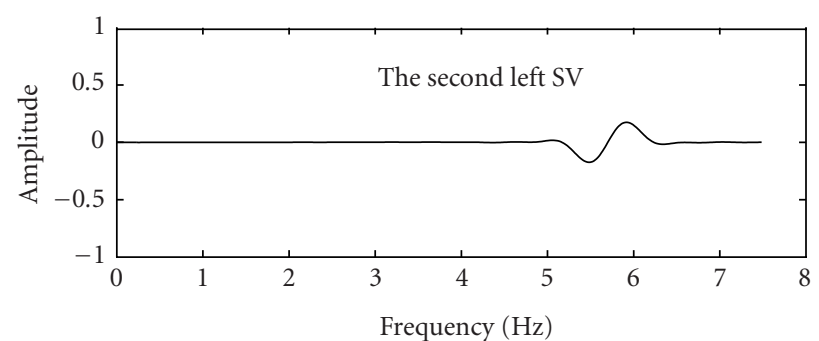

(b)

FIgURe 4: The first two left SVs of the TFDs related to (a) $x_{1}(t)$ and (b) $x_{2}(t)$.

The above examples show that to unambiguously characterise nonstationary signals in the TF domain, left and right SVs should be used simultaneously.

In [20], the authors introduced an SVD-based approach for TF feature extraction of nonstationary signals. The technique attempts to approximate the TF patterns through a number of rectangles. In the TF plot, the area with a uniform energy density is represented by a rectangle. The rectangles are identified by vectors of five elements $[\bar{t}, \bar{f}, \hat{t}, \hat{f}, \hat{\sigma}]$, where $\bar{t}$ and $\hat{t}$ represent the location and duration in time; $\bar{f}$ and $\hat{f}$ represent the location and width in frequency dimension in the TF plot; and $\hat{\sigma}$ represents the importance of the rectangle in the composition of the TF plot. The position and dimensions of the rectangles are computed from the first and second moments of the density functions extracted from the SVs of the TF plot.

The above-mentioned technique is useful for extracting features of nonstationary signals. However, it has three drawbacks. Firstly, it uses a fixed number of features (rectangles) to characterise the patterns embedded in TF plots. Using a limited number of rectangles may not be adequate to identify all possible patterns in the TF plot. Secondly, if there are more 


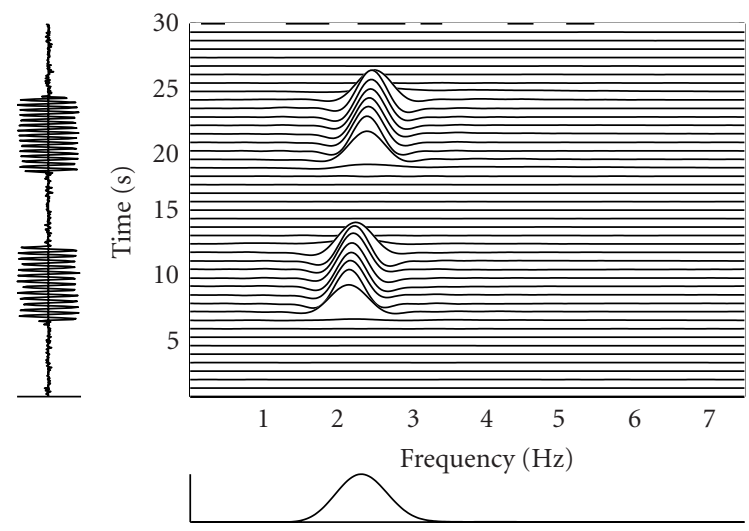

(a)
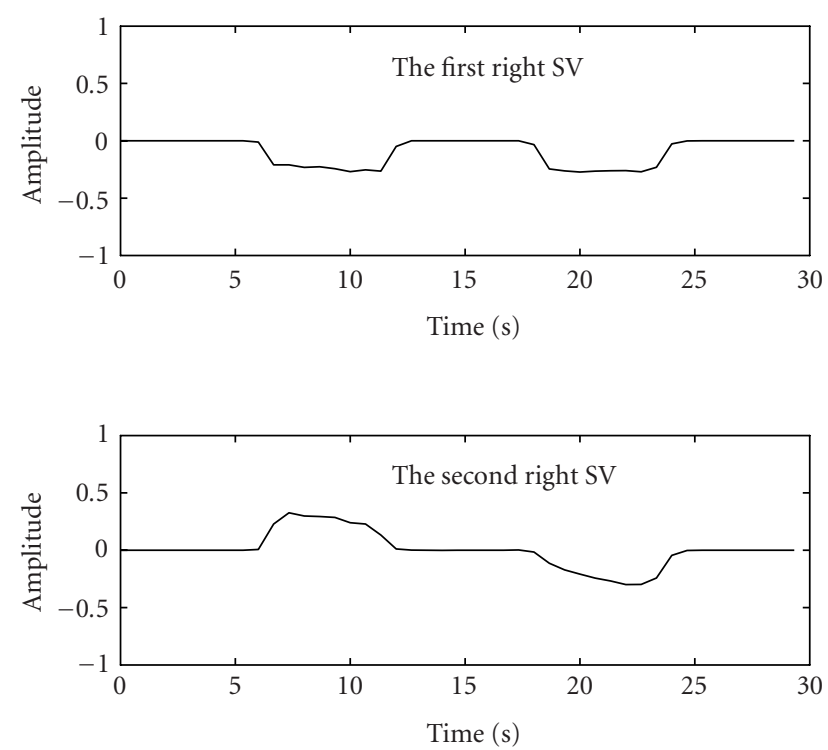

(c)

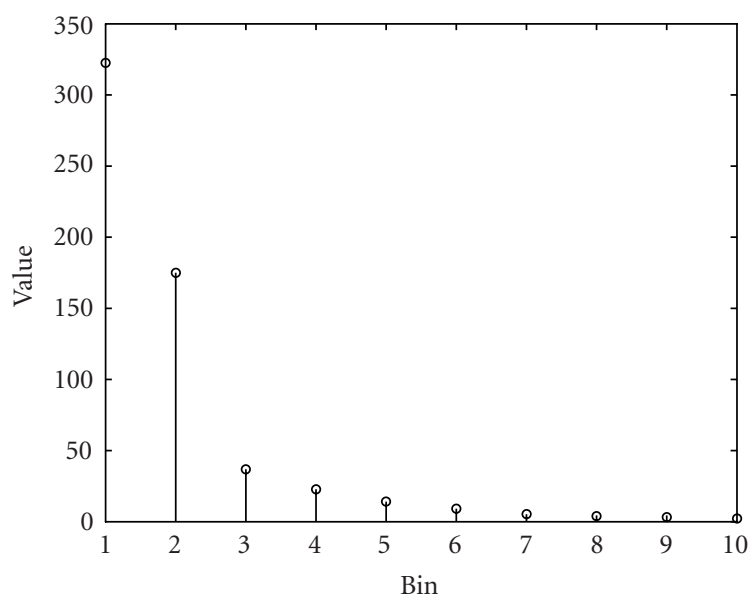

(b)
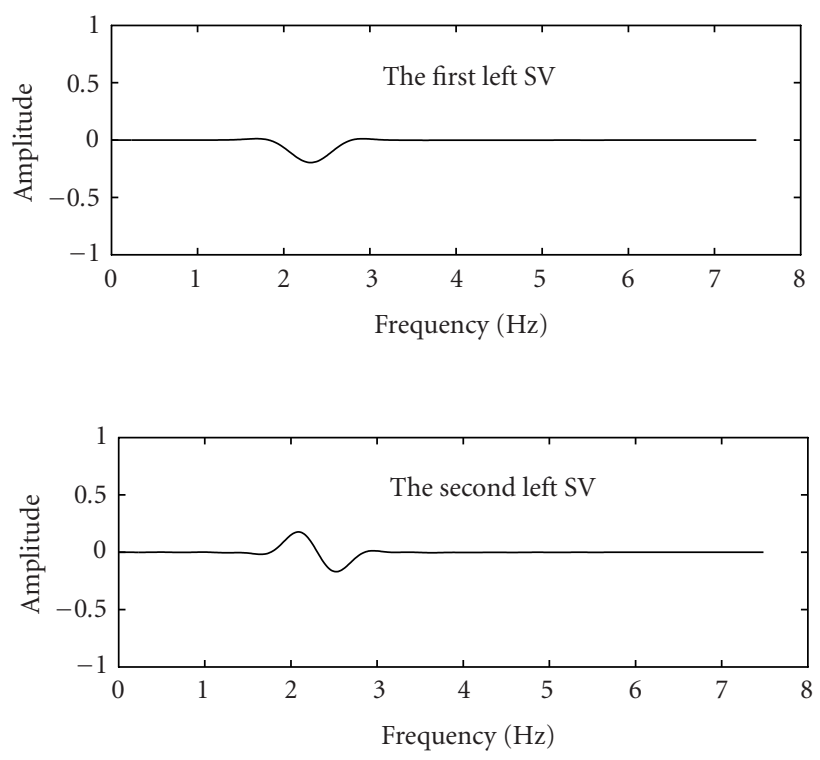

(d)

Figure 5: (a) The TFD of $x_{3}(t)(F s=15 \mathrm{~Hz}, N=450$, time resolution= 5). (b) Its singular values. ((c) and (d)) Its right and left SVs.

than one local maximum in the density function, the first and second moments of the density functions cannot show the position and the width of the local maxima. Hence, the technique may work well if (a) the TF patterns are simple enough to be approximated by a limited number of rectangles, and (b) the energy density of the signal is not uniformly concentrated at various locations of the TF plot. Thirdly, a TF pattern decomposed into the orthonormal bases, the left and right SVs, may not be addressed by only one left and right SVs. In other words, more than one left and right SVs may be needed to properly approximate a TF pattern. Hence, the moments extracted from only one left and right SVs are not enough to find the location, time duration, and frequency band of the pattern in the TF plot.
In another study [11], the same authors improved the feature extraction technique with respect to the third flaw. In this technique, the orthonormal bases created for a TF plot are rotated in order to minimise the number of vectors required in linear combinations to approximate the TF patterns.

\subsection{TF-based EEG seizure feature extraction}

Figure 6 shows the TFDs of two 30-second epochs of newborn EEG signal exhibiting seizure and nonseizure activities. The TFD were obtained using the B-distribution with parameter $\beta=0.01$. The SVD was applied to the TFD matrices to compute the left and right SVs. The two first left and right SVs are shown in Figures 7 and 8. 


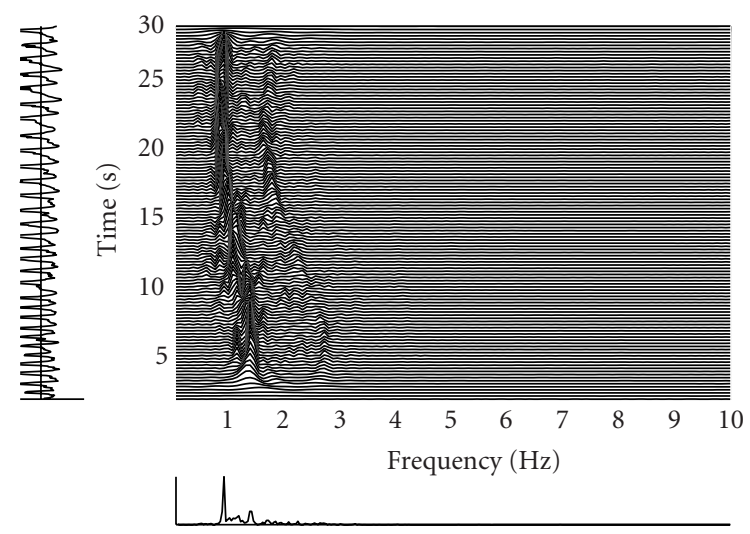

(a)

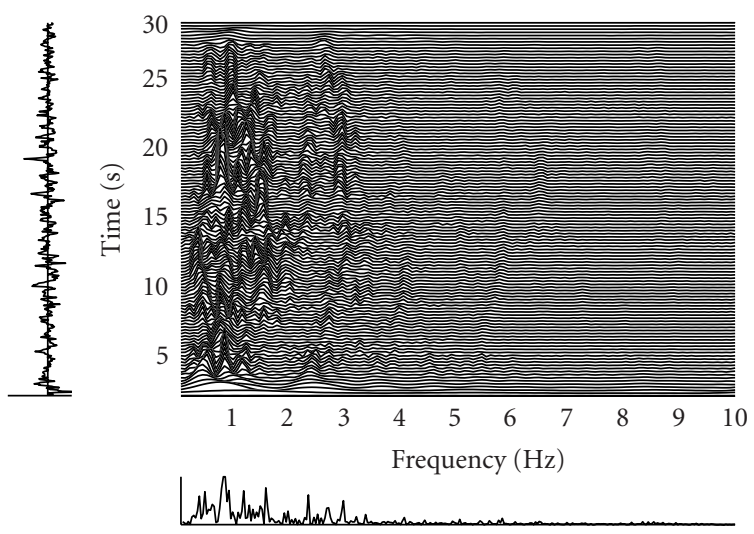

(b)

FIGURE 6: The TFD of two EEG epochs using the B-distribution: (a) seizure activity and (b) nonseizure activity $(F s=20 \mathrm{~Hz}, N=600$, time resolution=5).

In Figure 7, the first left SV shows that there is a burst of activity at frequencies around $1 \mathrm{~Hz}$, while the first right SV points to an activity that emerges 14 seconds after the beginning of the epoch and lasts about 15 seconds. The second left SV shows that there are high-energy activities around the frequencies $1.3 \mathrm{~Hz}$ and $1.5 \mathrm{~Hz}$. The second right SV indicates the presence of an activity that spans the whole 30-second epoch. These observations related to the first two SVs capture the essential information of the EEG seizure contained in the TF domain.

As shown above, a signal can be characterised by the SVs of its TFD. In other words, the SVs can be used as discriminating features in the seizure detection process. However, a reduced feature set with more appropriate features can provide a better classification accuracy with reduced data analysis cost [23]. In this paper, we suggest using a feature selection technique based on the probability distribution function of the SVs (DFSVs). This technique is described below.

Since the SVs are orthonormal, their squared elements can be treated as the different values of a PDF. The PDF can then be used to compute the probability distribution function.

Let $X_{t f}$ represents a TFD matrix of a signal $x$. Using SVD, this matrix can be represented as

$$
X_{t f}=U \Sigma V^{T},
$$

where $U(M \times M), \Sigma(M \times N)$, and $V(N \times N)$ are matrices of left SVs, singular values, and right SVs, respectively. The PDF can be formed from individual columns of matrices associated with the left and right SVs. For example, the PDF related to the first column of matrix $U, f_{U_{1}}$, is given by

$$
f_{U_{1}}=\left\{u_{11}^{2}, u_{12}^{2}, \ldots, u_{1 M}^{2}\right\}
$$

where $u_{1 i}$ represents the $i$ th element of $U_{1}$ (the first column of $U$ ), and $\sum_{i=1}^{M} u_{1 i}^{2}=1$. The related probability distribution function can be obtained as

$$
F_{U_{1}}=\left\{v_{1}, v_{2}, \ldots, v_{M}\right\}
$$

where

$$
v_{j}=\sum_{i=1}^{j} u_{1 i}^{2} \quad \text { for } j=1 \text { to } M .
$$

Distribution functions are nondecreasing, and it can be seen from Figures 9 and 10 that they have no significant changes in some areas. This is reflected in the corresponding histograms by few points with significant values. By using these histograms as features for detection, a considerable computational time will be gained.

Figures 9 and 10 show the distribution functions extracted from the first and second SVs associated with seizure and nonseizure activities shown in Figures 7 and 8, respectively. The histograms extracted from the left SVs show that for a signal including seizure, except the first and last bins, the content of the bins is almost zero.

\subsection{The feature extraction algorithm}

To summarise, the proposed TF-based algorithm for seizure feature extraction comprises the following steps.

Step 1. Filtering: since only the low-frequency signature of the seizure is of interest, any activity higher than $10 \mathrm{~Hz}$ is filtered.

Step 2. Segmentation: segmenting the EEG signal into 30second epochs without overlapping.

Step 3. Down sampling: reducing the sampling rate from 256 , the sampling rate in the recording time, to 20 samples per second to reduce the computational load. Following the Nyquist rate, this sampling rate is enough to analyse signals with frequencies less than $10 \mathrm{~Hz}$. The resample function of Matlab was used for the down-sampling process. 

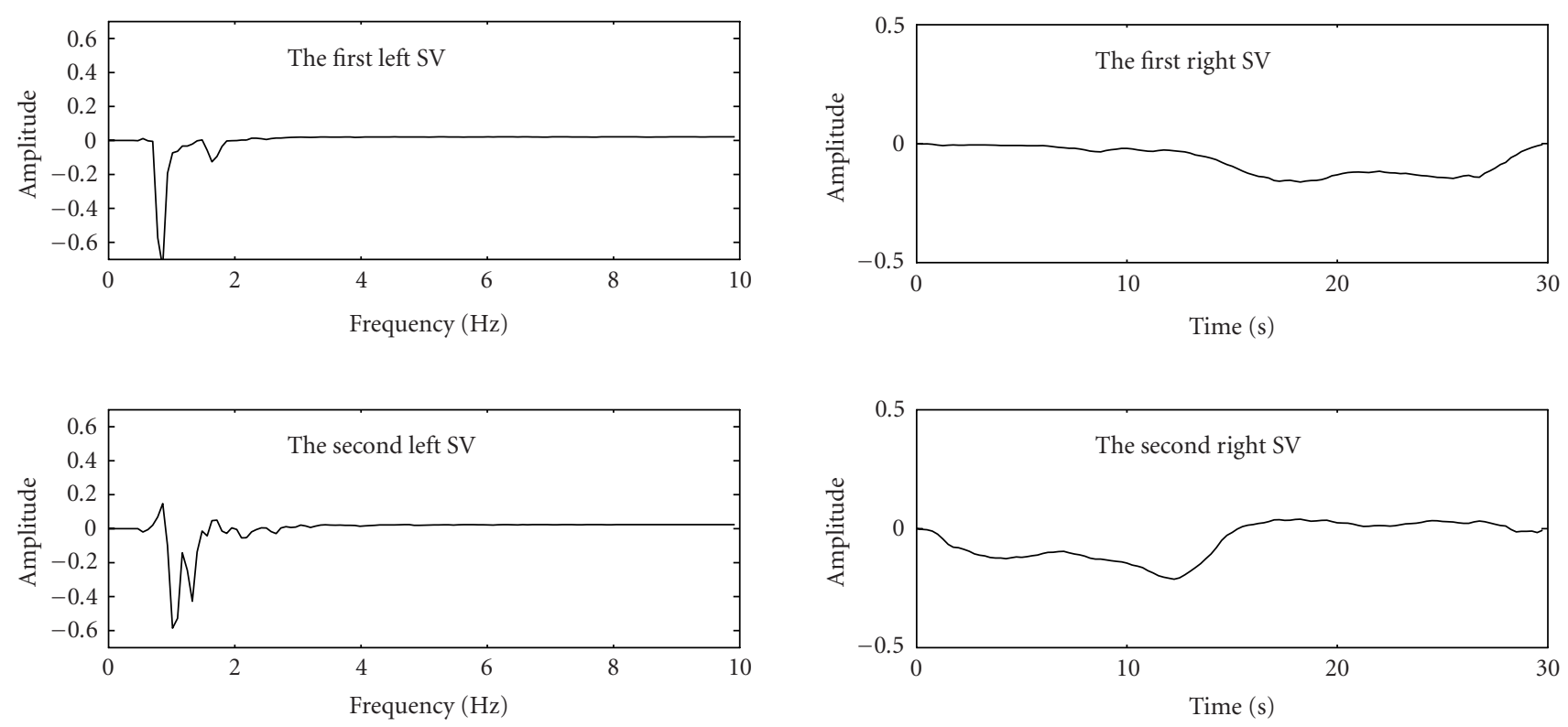

FIGURE 7: Left and right SVs of the matrix representing Figure 6a (seizure activity).
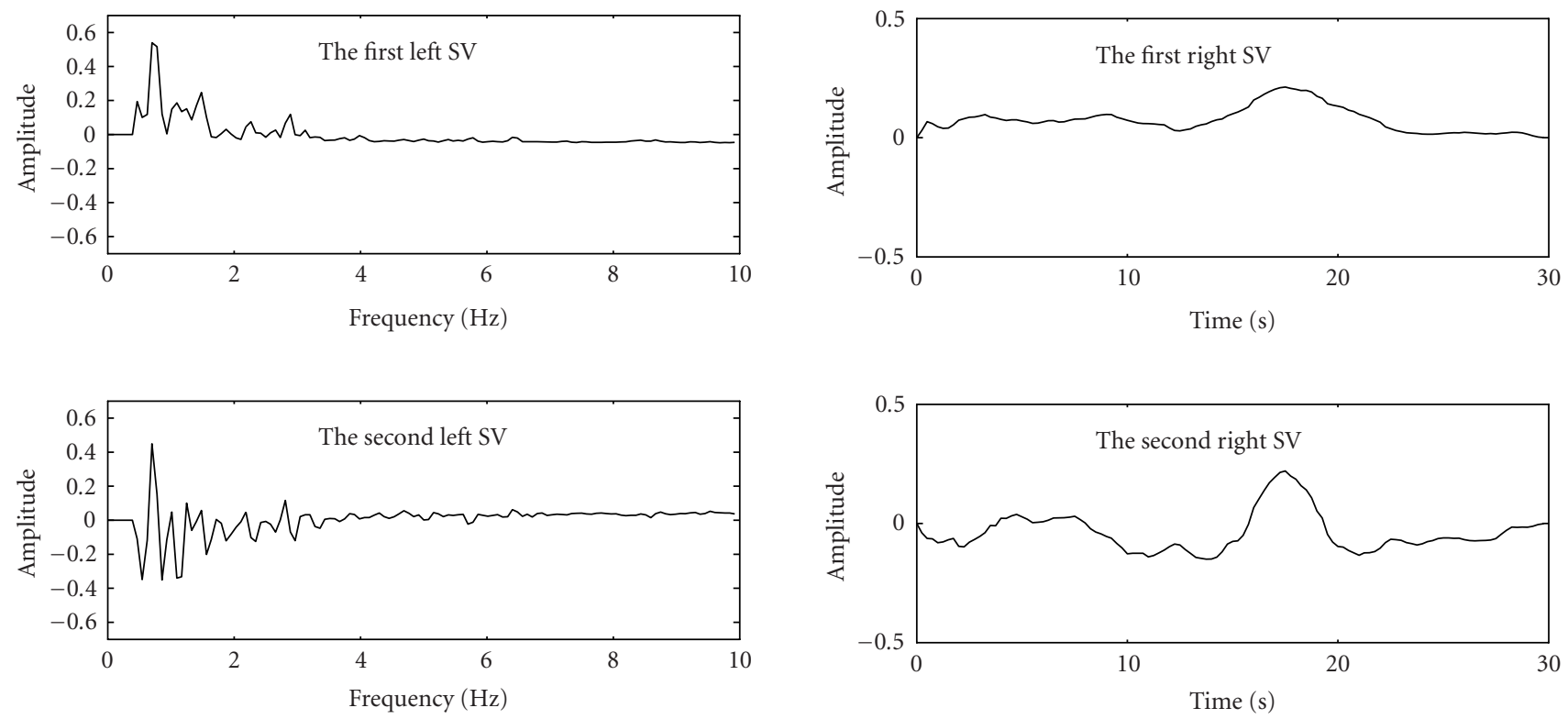

FIGURE 8: Left and right SVs of the matrix representing Figure 6b (nonseizure activity).

Step 4. TF representation: the 30-second EEG epoch is mapped into the TF domain using B-distribution with $\beta=$ 0.01 .

Step 5. Applying SVD: computing left and right SVs of the matrix related to the TF representation.

Step 6. Extracting distribution functions: since SVs are orthonormal, the squared elements of the SVs can be consid- ered as density functions. The density functions are then used for computing the distribution functions.

Step 7. Histogram computing: to compute the histogram related to the distribution function, we have used 11 bins. Successive bins have discrete elements of the distribution function with the maximum variation of 0.09 . Reducing the number of bins decreases performance of the system in differentiating between seizure and nonseizure activities. 

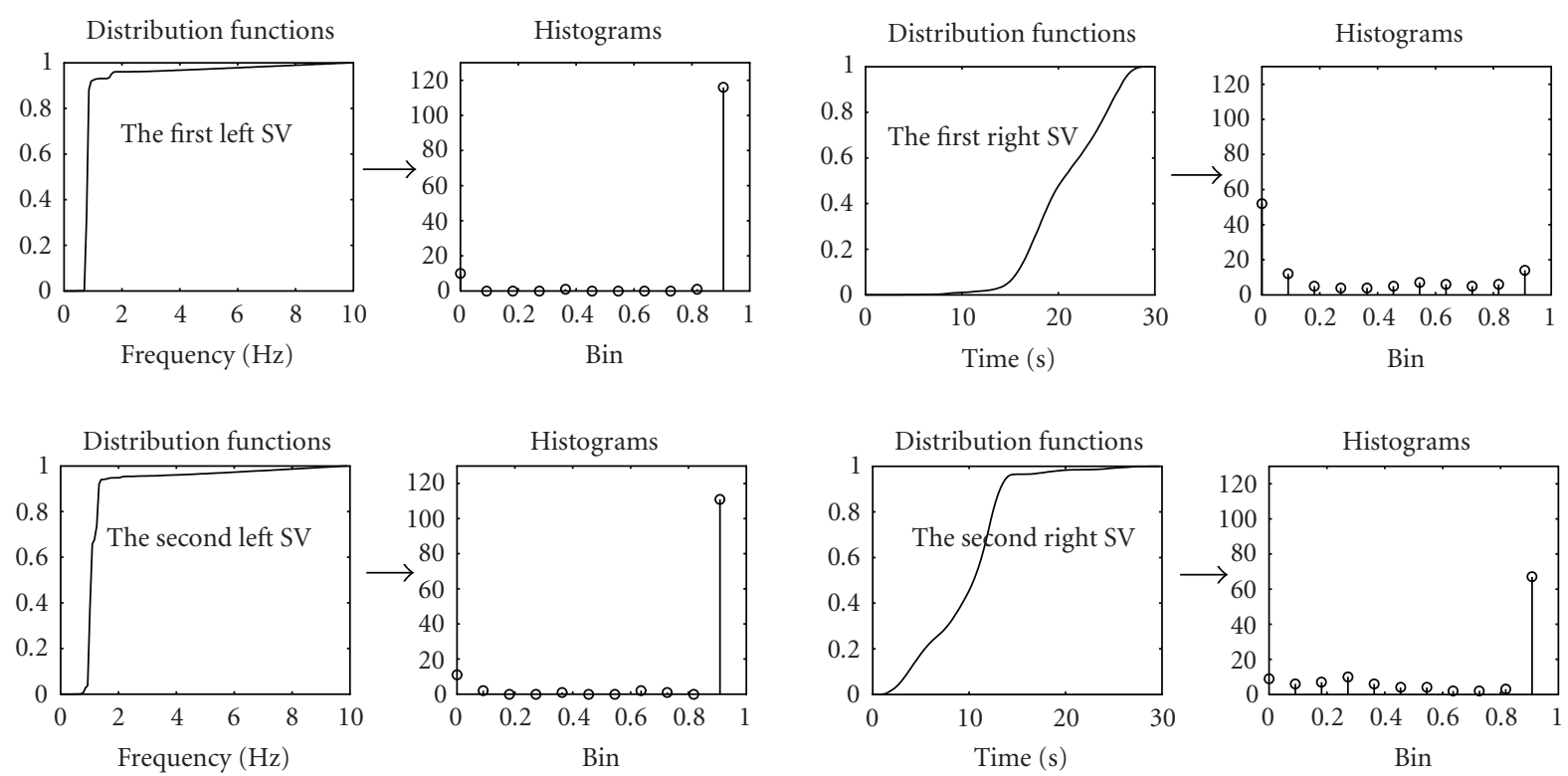

(a)

(b)

FIgURE 9: The probability distribution functions and their histograms associated with (a) the left SVs and (b) right SVs of the matrix representing Figure 6a (seizure activity).
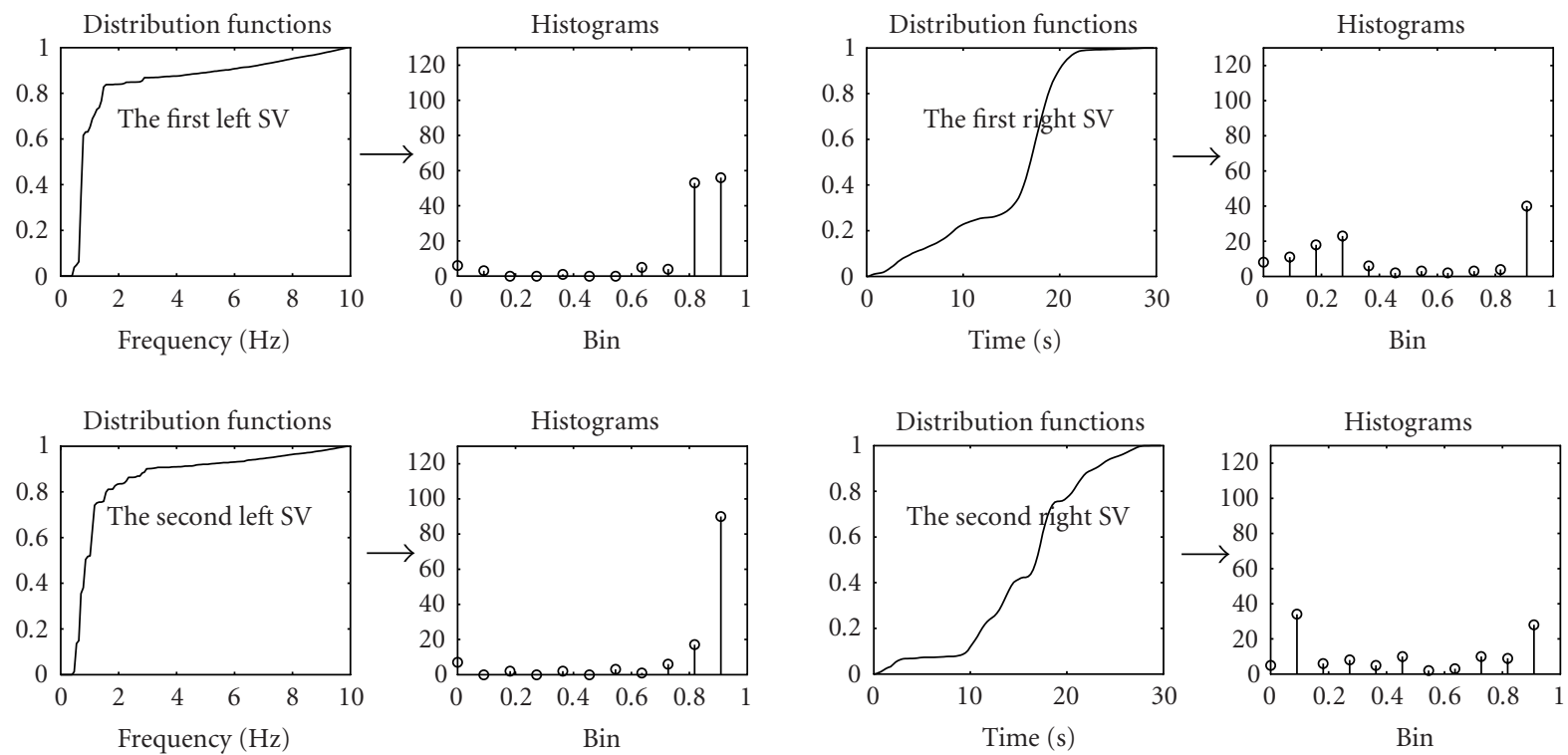

(a)

(b)

FIgURE 10: The probability distribution functions and their histograms associated with (a) the left SVs and (b) right SVs of the matrix representing Figure $6 \mathrm{~b}$ (nonseizure activity).

However, this number of bins was found to be adequate for 30-second epoch seizure detection.

\section{EEG SEIZURE DETECTION}

To discriminate between seizure and nonseizure activities in newborn EEG signals, we have used two left and two right
SVs related to the TFD of the 30-second EEG epoch. Experiments showed that using these vectors achieves good results. The feature extracted through the histogram of the four SVs was reorganised into a feature vector to be fed to a neural network. As the individual histograms have 11 bins, the length of the feature vector fed to neural network was 44. 
The neural network used in this research was a feedforward network. Networks with both one and two hidden layers using different neurons ( 2 to 15 neurons) in each of the hidden layers were studied. A two-layer neural network with 44,8 , and 2 neurons, respectively, in the input, hidden, and output layers offered the best detection rate. The network was then supervisely trained using the Levenberg-Marquardt algorithm [24].

\subsection{Experimental results and performance comparison}

In order to assess the performance of the above technique, the EEG data of eight newborns have been used. Firstly, we made a database of 30-second epochs associated with seizure and nonseizure activities. Seizure activities in the seizure epochs may have durations less than 30 seconds. The database includes 300 seizures and 800 nonseizures. To train the neural network, 200 seizures and 200 nonseizures were randomly selected and applied to the neural network. The training process learned the seizure and nonseizure patterns after 800 training iterations. The trained neural network was tested using the remaining EEG data and resulted in about $90 \%$ and $5.7 \%$ good detection rate (GDR)and false detection rate (FDR), respectively. The GDR and FDR are defined as

$$
\mathrm{GDR}=100 \times \frac{\mathrm{GD}}{R} \%, \quad \mathrm{FDR}=100 \times \frac{\mathrm{FD}}{\mathrm{GD}+\mathrm{FD}} \%,
$$

where GD and FD are the total number of good and false detections, respectively, and $R$ represents the total number of seizures recognised by the neurologist. A good detection occurs if the detected EEG epoch matches the epoch labeled as a seizure by the neurologist.

The performance of the proposed seizure detection method is compared with three other methods, namely, autocorrelation, spectrum, and singular spectrum analysis (SSA) techniques. These techniques are briefly described in the following sections.

\subsection{The autocorrelation technique}

The autocorrelation-based technique proposed by Liu et al. [25] relies on the assumption that the essential characteristic in newborn EEG seizures is periodicity. To asses the amount of periodicity, the EEG data is segmented into 30-second epochs and each epoch is divided into 5 windows. Depending on the autocorrelation function of a window, up to four primary periods are calculated for each window in an epoch. The windows are then scored whereby more evenly spaced primary periods are allocated larger scores. After each window in an epoch is scored, a rule-based detection scheme is applied to classify each epoch as seizure positive or negative. If two or more channels of EEG data in the same epoch are seizure positive, the epoch is then classified as containing seizure activity.

\subsection{The spectrum technique}

The method introduced by Gotman et al. was mainly based on the spectrum analysis of short epochs of EEG data
$[26,27]$. In this technique, to detect seizure activities, the EEG data is segmented into 10 -second epochs using a sliding window. The window is moved along the EEG in 2.5second steps. The algorithm was designed to extract features from each epoch and compare them with those of the background. The background is defined as a 20 -second segment of EEG finishing 60 seconds before the start of the current epoch. The main advantage of using a constantly updated background is that results are not dependent on the specific features of a fixed epoch.

The frequency spectrum of the individual epochs is calculated and the following features are extracted: (1) the frequency of the dominant spectral peak, (2) the width of the dominant spectral peak, and (3) the ratio of the power in the dominant spectral peak to that of the background spectrum in the same frequency band.

The three features are used in detecting seizures in each epoch. If an epoch is recognised as containing seizure, a further three criteria are employed to reduce the rate of false detections. Detected seizures are ignored if the epoch is largely nonstationary, if there is a large amount of AC power noise present, or if it appears that an EEG lead has been disconnected.

The aim of this technique is to determine whether a dominant peak exists in the power spectral density estimate. This is equivalent to finding whether an EEG signal has a dominant periodic shape in the time domain. The features used to classify an epoch as a seizure ensure that the dominant peak of the spectrum is significant compared with the background spectrum.

\subsection{The SSA technique}

Celka et al [7] proposed a method for newborn EEG seizure detection using SSA. The SSA method is suited for extracting information from stationary or at least quasistationary signals cluttered with noise.

In this method, to detect seizure activity in EEG data, the signal is preprocessed. The preprocessing is based on a nonlinear whitening filter that spreads the spectrum of the background while keeping rhythmical features of the seizure activities. The filtered signal is then segmented into 10 -second epochs using sliding window with 1.25-second steps. The individual epochs are converted into a matrix for separating the noise subspace from the signal subspace. The signal subspace is obtained by using $n_{0}$ SVs related to the $n_{0}$ largest singular values of the matrix using the SVD technique. To find $n_{0}$, as a criterion for space division, they used the Rissanen minimum description length (MDL) method. In this technique, if $n_{0}$ is equal to 1 , the related epoch is considered as a background; otherwise, it is a seizure.

\subsection{Performance comparison and discussion}

The performance assessment of the above-mentioned methods was accomplished by applying their algorithms to all the EEG channels of each newborn. In using the DFSV technique, the EEG epoch is considered to contain a seizure in 
TABLE 1: Performance comparison of the DFSV with the three other methods.

\begin{tabular}{l|cccccccc}
\hline \multirow{2}{*}{ Patients } & \multicolumn{2}{|c}{ Autocorrelation } & \multicolumn{2}{c}{ Spectral } & \multicolumn{2}{c}{ SSA } & \multicolumn{2}{c}{ DFSV } \\
& GDR & FDR & GDR & FDR & GDR & FDR & GDR & FDR \\
\hline Baby 1 & $50 \%$ & $11 \%$ & $44 \%$ & $14 \%$ & $50 \%$ & $19 \%$ & $66 \%$ & $9 \%$ \\
Baby 2 & $32 \%$ & $7.5 \%$ & $47 \%$ & 0 & $97 \%$ & $2 \%$ & $98 \%$ & $2 \%$ \\
Baby 3 & $95 \%$ & $37 \%$ & $85 \%$ & $36 \%$ & $99 \%$ & $35 \%$ & $95 \%$ & $15 \%$ \\
Baby 4 & $31 \%$ & 0 & 0 & 0 & $91 \%$ & 0 & $96 \%$ & 0 \\
Baby 5 & $67 \%$ & $1 \%$ & $78 \%$ & $1 \%$ & $98 \%$ & $1 \%$ & $99 \%$ & $1 \%$ \\
Baby 6 & $29 \%$ & $9 \%$ & 0 & 0 & $87 \%$ & 0 & $92 \%$ & 0 \\
Baby 7 & $72 \%$ & $3 \%$ & $33 \%$ & 0 & $97 \%$ & $2 \%$ & $95 \%$ & $2 \%$ \\
Baby 8 & $77 \%$ & $1 \%$ & $67 \%$ & 0 & $97 \%$ & $1 \%$ & $99 \%$ & $1 \%$ \\
\hline Average & $56.6 \%$ & $8.6 \%$ & $44.2 \%$ & $6.3 \%$ & $89.5 \%$ & $7.5 \%$ & $92.5 \%$ & $3.7 \%$ \\
\hline
\end{tabular}

a given time interval if the algorithm detects a seizure in one or more channels in that specific interval. The performance results are summarised in Table 1. The results show that the DFSV technique has the overall better performance than the other techniques in terms of the GDR and FDR. For Baby 3, although the DFSV has 4\% lower GDR than the SSA, its FDR is remarkably lower than all the other tested techniques. The GDRs of all four techniques for Baby 1 are considerably lower than those of the other babies. The reason could be the lack of low-frequency signature of seizures as all of the techniques are based on the lowfrequency signatures. In such case, EEG seizures can be detected using the high-frequency signature as mentioned in [28].

\section{CONCLUSIONS}

This paper presents a new TF-based technique for detecting seizure activity in the EEG signal of neonates. The detection process uses the low-frequency signature of seizures. To detect EEG seizure, the signal is segmented into 30-second epochs and analysed using the SVs of the TFD of the signal. Histograms extracted from the distribution function formed from the squared-elements of the left and right SVs were shown to efficiently discriminate between the seizure and nonseizure activities as evidenced by the high detection rates. The GDR resulted from applying the untrained data set to the neural network shows the good quality of the extracted feature.

This technique is based on low-frequency signature of the seizures. In a related work, we have shown that some types of seizures may have only signatures in highfrequency area. This fact may potentially result in a reduction of the seizure detection rate. To overcome this problem, the authors proposed a new technique based on high-frequency seizure signature and are working toward a method that can effectively combine the detectors based on those types of signatures. The results of the work will appear elsewhere.

\section{ACKNOWLEDGMENTS}

This research is funded by the Australian Research Council (ARC). The authors wish to thank Professor Paul Colditz of the Royal Women's Hospital in Brisbane for providing access to the Perinatal Research Centre and Dr. Chris Burke of the Royal Children's Hospital in Brisbane for his assistance in the interpretation of the EEG data.

\section{REFERENCES}

[1] E. M. Mizrahi and P. Kellaway, "Neonatal electrocephalography," in Diagnosis and Management of Neonatal Seizures, pp. 99-143, Lippincott-Raven, Philadelphia, Pa, USA, 1998.

[2] P. L. Paige and P. R. Carney, "Neurologic disorders," in Handbook of Neonatal Intensive Care, chapter 25, pp. 644-678, Mosby, St. Louis, Mo, USA, 2002.

[3] C. T. Lombroso, "Neonatal EEG polygraphy in normal and abnormal newborns," in Electroencephalography. Basic Principles, Clinical Applications and Related Fields, E. Niedermeyer and F. H. Lopes da Silva, Eds., chapter 48, pp. 803-875, Williams and Wilkins, Baltimore, Md, USA, 1993.

[4] E. M. Mizrahi, P. Plouin, and P. Kellaway, "Neonatal seizures," in Epilepsy: A Comprehensive Textbook, J. Engel and T. A. Pedley, Eds., vol. 1, chapter 57, pp. 647-663, Lippincott-Raven, Philadelphia, Pa, USA, 1997.

[5] P. Celka and P. Colditz, "Nonlinear nonstationary wiener model of infant EEG seizures," IEEE Transactions on Biomedical Engineering, vol. 49, no. 6, pp. 556-564, 2002.

[6] B. Boashash, H. Carson, and M. Mesbah, "Detection of seizures in newborns using time-frequency analysis of EEG signal," in Proc. 10th IEEE Workshop on Statistical Signal and Array Processing, pp. 564-568, Pocono Manor, Pa, USA, August 2000.

[7] P. Celka and P. Colditz, "A computer-aided detection of EEG seizures in infants: a singular-spectrum approach and performance comparison," IEEE Transactions on Biomedical Engineering, vol. 49, no. 5, pp. 455-462, 2002.

[8] B. Boashash and M. Mesbah, "A time-frequency approach for newborn seizure detection," IEEE of EMBS Magazine, vol. 20, no. 5, pp. 54-64, 2001.

[9] H. Hassanpour, W. J. Williams, M. Mesbah, and B. Boashash, "Time-frequency extraction of EEG spike events for seizure detection in neonate," in Proc. 6th International Symposium on Signal Processing and Its Application (ISSPA '01), vol. 1, pp. 246-249, Kuala Lumpur, Malaysia, August 2001. 
[10] G. Nakos and D. Joyner, Linear Algebra with Applications, Brooks/Cole Publishing Company, Pacific Grove, Calif, USA, 1998.

[11] D. Groutage and D. Bennink, "A new matrix decomposition based on optimum transformation of the singular value decomposition basis sets yields principal features of timefrequency distributions," in Proc. 10th IEEE Workshop on Statistical Signal and Array Processing, pp. 598-602, Pocono Manor, Pa , USA, August 2000.

[12] L. Cohen, "The need for time-freaquency analysis," in Time-Frequency Analysis, Prentice Hall Signal Processing Series, chapter 5, pp. 70-81, Prentice-Hall, Englewood Cliffs, NJ, USA, 1995.

[13] S. S. Abeysekera and B. Boashash, "Methods of signal classification using the images produced by the Wigner-Ville distribution," Pattern Recognition Letters, vol. 12, no. 11, pp. 717729, 1991.

[14] B. Boashash, "Part I: Introduction to the concepts of TFSAP," in Time-Frequency Signal Analysis and Processing: A Comprehensive Reference, pp. 1-81, Elsevier, Oxford, UK, 2003.

[15] B. Boashash and B. Barkat, "Introduction to time-frequency signal analysis," in Wavelet Transforms and Time-Frequency Signal Analysis, L. Debnath, Ed., pp. 321-380, Birkhäuser, Boston, Mass, USA, 2000.

[16] W. J. Williams, "Reduced interference time-frequency distributions," in Time-Frequency Signal Analysis and Processing: A Comprehensive Reference, B. Boashash, Ed., chapter 5, pp. 168-177, Elsevier, Oxford, UK, 2003.

[17] B. Barkat and B. Boashash, "A high-resolution quadratic timefrequency distribution for multicomponent signals analysis," IEEE Trans. Signal Processing, vol. 49, no. 10, pp. 2232-2239, 2001.

[18] N. M. Marinovic and G. Eichmann, "Feature extraction and pattern classification in space-spatial frequency domain," in SPIE Intelligent Robots and Computer Vision, vol. 579 of Proceedings of SPIE, pp. 19-25, September 1985.

[19] S. Gu, J. Ni, and J. Yuan, "Non-stationary signal analysis and transient machining process condition monitoring," International Journal of Machine Tools and Manufacture, vol. 42, no. 1, pp. 41-51, 2002.

[20] D. Groutage and D. Bennink, "Feature sets for nonstationary signals derived from moments of the singular value decomposition of Cohen-Posch (positive time-frequency) distributions," IEEE Trans. Signal Processing, vol. 48, no. 5, pp. 1498 1503,2000

[21] V. Venkatachalam and J. L. Aravena, "Nonstationary signal classification using pseudo power signatures: The matrix SVD approach," IEEE Trans. on Circuits and Systems II: Analog and Digital Signal Processing, vol. 46, no. 12, pp. 1497-1505, 1999.

[22] D. Bernal, "Damage localization using load vectors," in European COST F3 Conference, pp. 223-231, Madrid, Spain, June 2000.

[23] A. Jain and D. Zongker, "Feature selection: evaluation, application, and small sample performance," IEEE Trans. on Pattern Analysis and Machine Intelligence, vol. 19, no. 2, pp. 153-158, 1997.

[24] G. Lera and M. Pinzolas, "Neighborhood based LevenbergMarquardt algorithm for neural network training," IEEE Transactions on Neural Networks, vol. 13, no. 5, pp. 12001203, 2002.

[25] A. Liu, J. S. Hahn, G. P. Heldt, and R. W. Coen, "Detection of neonatal seizures through computerized EEG analysis," Electroencephalography and Clinical Neurophysiology, vol. 82, no. 1, pp. 30-37, 1992.

[26] J. Gotman, D. Flanagan, J. Zhang, and B. Rosenblatt, "Automatic seizure detection in the newborn: methods and initial evaluation," Electroencephalography and Clinical Neurophysiology, vol. 103, no. 3, pp. 356-362, 1997.

[27] J. Gotman, J. Zhang, B. Rosenblatt, and R. Gottesman, "Automatic seizure detection in newborns and infants," in Proc. IEEE 17th Annual Conference on Engineering in Medicine and Biology Society, vol. 2, pp. 913-914, Montreal, Quebec, Canada, September 1995.

[28] H. Hassanpour and M. Mesbah, "Neonatal EEG seizure detection using spike signatures in the time-frequency domain," in Proc. International Symposium on Signal Processing and Its Application (ISSPA '03), vol. 2, pp. 41-44, Paris, France, July 2003.

Hamid Hassanpour received the B.S. degree in computer engineering from Iran University of Science \& Technology, Tehran, Iran, in 1994, the M.S. degree in computer engineering from Amirkabir University of Technology, Tehran, Iran, in 1997, and the Ph.D. degree in signal processing from Queensland University of Technology, Brisbane, Australia. His research interests include signal detection and classification, biomedical signal processing, and time-frequency signal analysis.

Mostefa Mesbah received his M.S. and Ph.D. degrees in electrical engineering from University of Colorado at Boulder, Colorado, USA, in the area of automatic control systems. He is currently a Senior Researcher at the Signal Processing Research Centre (SPRC), Queensland University of Technology in Brisbane, Australia, leading a biomedical engineering project that deals with the automatic detection and classification of newborn EEG seizures. His research interests include biomedical signal processing, time-frequency signal processing, signal detection and classification, 3D shape reconstruction from image sequences, and intelligent control systems.

Boualem Boashash obtained a Diplome d'ingenieur-Physique-Electronique from Institut de Chimie et de Physique Industrielles de Lyon (ICPI), University of Lyon, France, in 1978, the M.S. and Doctorate (Docteur-Ingenieur) degrees from the Institut National Polytechnique de Grenoble, France, in 1979 and 1982, respectively. In 1979, he joined Elf-Aquitaine Geophysical Research Centre, Pau, France. In May 1982, he joined the Institut National des Sciences Appliquées de Lyon, France. In 1984, he joined the Electrical Engineering Department, University of Queensland, Australia, as a Lecturer. In 1990, he joined Graduate School of Science and Technology, Bond University, as a Professor of electronics. In 1991, he joined Queensland University of Technology as the foundation Professor of signal processing and Director of the Signal Processing Research Centre. B. Boashash is the Editor of three books and has written over four hundred technical publications. His research interests include time-frequency signal analysis, spectral estimation, signal detection and classification, and higher-order spectra. Professor Boashash is a Fellow of Engineers Australia, Fellow of IREE, and Fellow of IEEE. 